\title{
A gastrin transcript expressed in gastrointestinal cancer cells contains an internal ribosome entry site
}

\author{
AM Grabowska*,', CA Berry', J Hughes', M Bushell', AE Willis² and SA Watson' \\ 'Division of Pre-Clinical Oncology, School of Medical and Surgical Sciences, University of Nottingham, Nottingham, UK; ${ }^{2}$ School of Pharmacy, Centre for \\ Biomolecular Sciences, University of Nottingham, Nottingham, UK
}

As the hormone gastrin promotes gastrointestinal (Gl) cancer progression by triggering survival pathways, regulation of gastrin expression at the translational level was explored. Sequence within the $5^{\prime}$ untranslated region of a gastrin transcript expressed in $\mathrm{Gl}$ cancer cells was investigated, then cloned into a bicistronic vector upstream of firefly luciferase and transfected into a series of Gl cancer cell lines. Firefly luciferase activity was measured relative to that of a cap-dependent Renilla luciferase. A gastrin transcript that was different from that described in Ensembl was expressed in $\mathrm{Gl}$ cancer cells. Its transcription appears to be initiated within the region designated as the gene's first intron. In Gl cancer cells transfected with the bicistronic construct, firefly luciferase activity increased 8-15-fold compared with the control vector, and there was a further induction of the signal (up to 25-fold) following exposure of the cells to genotoxic stress or hypoxia, suggesting that the sequence acts as an internal ribosome entry site. These data suggest that the gastrin transcript within Gl cancer cells contains an internal ribosome entry site that may allow continued expression of gastrin peptides when normal translational mechanisms are inactive, such as in hypoxia, thereby promoting cancer cell survival. British Journal of Cancer (2008) 98, 1696- 1703. doi:I0.I038/sj.bjc.6604326 www.bjcancer.com

Published online 8 April 2008

(c) 2008 Cancer Research UK

Keywords: gastrin; translation; gastrointestinal; internal ribosome entry

Gastrin is normally expressed in G cells of the stomach antrum and regulates both acid secretion and proliferation of gastric mucosal cells (Watson et al, 2006). The gastrin gene (GAST) is a 4-kb unit consisting of three exons and two introns with the gastrin polypeptide encoded by sequence within exons 2 and 3 (http:// www.ensembl.org/index.html). Two different gastrin transcripts have been described in the literature. The transcript given in the Ensembl database was described in human gastrinomas and is a 434-bp transcript incorporating sequence from exon 1 (Ito et al, 1984; Wiborg et al, 1984). However, another transcript was identified in the gastric antrum that has a transcription start site $111 \mathrm{bp}$ upstream of the start codon (Kato et al, 1983). Thus, the $5^{\prime}$ untranslated regions ( $5^{\prime}$ UTRs) of the Ensembl and alternative transcripts are different.

Gastrin upregulation has been shown at both the gene and protein levels in a number of gastrointestinal (GI) (Goetze et al, 2000; Mukawa et al, 2005; Hur et al, 2006) and non-GI cancers (Rehfeld et al, 1989; van Solinge et al, 1993). At the transcriptional level in GI cancer, gastrin upregulation may be a result of mutational events, for example in the APC (adenomatous polyposis coli) or $k$-ras genes (Nakata et al, 1998; Koh et al, 2000); engagement of the EGF (epidermal growth factor) receptor

*Correspondence: Dr AM Grabowska, Division of Pre-Clinical Oncology, University of Nottingham, D Floor, West Block, Queens Medical Centre, Nottingham NG7 2UH, UK;

E-mail: anna.grabowska@nottingham.ac.uk

Received 31 August 2007; revised 28 February 2008; accepted 3 March 2008; published online 8 April 2008
(Merchant et al, 1991); inflammatory events mediated directly by cytokines, such as those associated with Helicobacter pylori infection (Suzuki et al, 2001; Beales, 2004); or direct activation by certain pathogenicity factors expressed by Helicobacter pylori (Rieder et al, 2005).

Expression of a number of genes that promote cancer cell survival has been shown to be regulated at the translational level (Pickering and Willis, 2005; Sontheimer and Carthew, 2005). One mechanism, involving the presence of an internal ribosome entry site (IRES) within the $5^{\prime} \mathrm{UTR}$ of the transcript, may have evolved to allow continued expression of key proteins involved in cell survival during cellular stress (Bushell et al, 2004; Holcik and Sonenberg, 2005) when conventional cap-mediated translation is reduced. However, it may also contribute to cancer cell survival as IRESs have been identified in the transcripts of genes that increase proliferation, protect against apoptosis and promote angiogenesis (Vagner et al, 1995; Miller et al, 1998; Stoneley et al, 2000a; Coldwell et al, 2001).

Gastrin plays an important role in establishing and supporting the growth of a range of GI tumours (Ferrand and Wang, 2006; Watson et al, 2006). As well as acting as a growth hormone, it has well-documented pro-angiogenic (Clarke et al, 2006) and antiapoptotic properties (Konturek et al, 2003; Harris et al, 2004; Ramamoorthy et al, 2004). We have previously used RNAi to downregulate gastrin expression at the gene level and observed a rapid loss of the transcript, but also a delayed downregulation of the endogenous protein compared with GFP-tagged gastrin encoded by a transcript lacking the gastrin $5^{\prime} \mathrm{UTR}$ (Grabowska et al, 2007). This raised the possibility that gastrin expression may be regulated translationally in a manner dependent on the $5^{\prime} \mathrm{UTR}$ 
allowing continued protein expression from the small amount of transcript remaining in the cells following RNAi-mediated knockdown. Therefore, we investigated the $5^{\prime} \mathrm{UTR}$ of the gastrin transcript for sequences that might regulate gastrin expression at the translational level in GI cancer.

\section{MATERIALS AND METHODS}

\section{Cell culture}

PAN1 is a human pancreatic cell line derived from a poorly differentiated human pancreatic adenocarcinoma within the Division of Pre-Clinical Oncology, University of Nottingham (UK). HCT116, a poorly differentiated human colon cell line, was obtained from ECACC (reference no. 91091005). All cell lines were routinely cultured in RPMI 1640 culture medium (Gibco, Paisley, UK) containing $10 \%(\mathrm{v} / \mathrm{v})$ heat-inactivated fetal bovine serum (FBS; Sigma, Poole, UK) at $37^{\circ} \mathrm{C}$ in $5 \% \mathrm{CO}_{2}$ and humidified conditions.

\section{RNA ligase-mediated rapid amplification of cDNA ends}

RNA was extracted from PAN1 cells using RNABee (Biogenesis, Poole, UK) and quantified using an ND-100 Spectrophotometer (Nanodrop, Wilmington, USA). Analysis of the $5^{\prime}$ ends of the gastrin transcripts was carried out using the RLM-RACE kit, according to the manufacturer's instructions (Ambion, Cambs, UK). The sequences of the primers used are given in Table 1 . Briefly, $3 \mu \mathrm{g}$ of total RNA was treated with calf intestine alkaline phosphatase for $1 \mathrm{~h}$ at $37^{\circ} \mathrm{C}$ (to remove free $5^{\prime}$ phosphate groups), phenol-chloroform extracted, isopropanol precipitated, then treated with Tobacco Acid Pyrophosphatase for $1 \mathrm{~h}$ at $37^{\circ} \mathrm{C}$ to remove the $5^{\prime}$ cap. A rapid amplification of cDNA ends (RACE) adapter was ligated to the product using T4 RNA ligase, and cDNA synthesis carried out using random decamers and M-MLV Reverse Transcriptase. A nested polymerase chain reaction (PCR) was carried out using the cDNA as a template. In the first round, the $5^{\prime}$ RACE outer primer was used with the gastrin RACE outer primer. In the second round, the $5^{\prime}$ RACE inner primer was used with the gastrin RACE inner primer.

\section{TA cloning}

The PCR products from the RNA ligase-mediated rapid amplification of cDNA ends (RLM-RACE) nested PCR were cloned into pCRII-TOPO using the TOPO TA cloning kit (Invitrogen, UK) and transformed into $\mathrm{TOP} 10 \mathrm{~F}^{\prime}$ competent cells (Invitrogen). Clones containing inserts were selected by blue-white screening and DNA

Table I Primer sequences

\begin{tabular}{ll}
\hline Primer & Sequence \\
\hline RACE adapter & GCUGAUGGCGAUGAAUGAACACUGCGUUU \\
& GCUGGCUUUGAUGAA \\
5' RACE outer primer & GCTGATGGCGATGAATGAACACTG \\
Gastrin RACE outer primer & TCCAGCCAGGGTAGCTCCAG \\
5' RACE inner primer & CGCGGATCCGAACACTGCGTTTGCTGGCT \\
& TTGATG \\
Gastrin RACE inner primer & GGCTTCCAAGAAGCTTCAGA \\
GasEnsF & CCACACACCTCCCAGCTC \\
GasAltF & TGGAGCCACATGGTTCAGT \\
HGASL & CCCATCCATCCATAGGCTTC \\
EcoRI GasIRESF & ACACACACAGTCGCTCCAtggCGTCTGCAA \\
Ncol GasIRESR & GCAAGAAGATGCACCTGATG \\
Renilla luciferase F & GCGTATCTCTTCATAGCCTT \\
Firefly luciferase R & \\
\hline
\end{tabular}

prepared using Genelute plasmid miniprep kits (Sigma). The DNA was sequenced using a T7 primer and BigDye reaction mix and analysed using an ABI 310 Genetic Analyser (ABI, Warrington, UK). Similarity between the RLM-RACE products and the gastrin gene and transcript sequences obtained from Ensembl Genome Browser (http://www.ensembl.org/index.html) was compared using the ClustalW program in Biology Workbench (http:// workbench.sdsc.edu/). The sequence was also submitted to UTRScan (http://www.ba.itb.cnr.it/BIG/UTRScan/).

\section{Reverse transcriptase-PCR (RT-PCR)}

To detect expression of gastrin transcripts, RNA was extracted using the PARIS mirVana kit (Ambion) using the protocol for 'large' RNA, then reverse transcribed into cDNA using the Quantitect Reverse Transcription kit (Qiagen, Sussex, UK), which includes a step for removal of genomic contamination. For each RNA, a duplicate reaction was carried out omitting the reverse transcriptase (RT) to ensure that all genomic contamination had been removed. A $2 \mu \mathrm{l}$ aliquot of the cDNA or 'RT minus' control was amplified using a primer specific for the Ensembl transcript (GasEnsF) or the alternative transcript (GasAltF) in combination with a common reverse primer (HGASL). The sequences of the primers are given in Table 1. A $10 \mu \mathrm{l}$ aliquot of the PCR reaction was analysed on a $2 \%$ agarose gel alongside 2-log markers (New England Biolabs, Herts, UK) and visualised using ethidium bromide staining.

To investigate the size of transcripts synthesised from the bicistronic constructs, RNA was prepared using RNABee as described above and cDNA synthesised using Superscript II and random hexamers as described previously (McWilliams et al, 1998). To ensure that signals generated were derived from RNA, RT-negative, cDNA reagent and extraction controls were included in which the RT was omitted from the cDNA synthesis, RNA was omitted from the cDNA synthesis or cells were omitted from the RNA extraction. Polymerase chain reaction was carried out using forward and reverse primers located within the Renilla and firefly luciferases, respectively, to determine the size of the transcript generated from the plasmid. The sequences of the primers are given in Table 1.

\section{Reporter construct cloning}

The putative gastrin IRES was PCR amplified using forward and reverse primers (EcoRI GasIRESF and NcoI GasIRESR) designed to contain EcoRI and NcoI restriction enzyme sites, respectively (shown in lower case in the primer sequences given in Table 1) to facilitate cloning. In the reverse primer, the NcoI site was designed to lie over the gastrin start codon, which involved making mutations in the sequence (shown in Table 1 by underlining of altered nucleotides) to create the restriction enzyme site. Following restriction digestion of the PCR product with EcoRI and NcoI, the fragment was cloned into the bicistronic reporter construct, $\mathrm{pRF}$ (Stoneley et al, 2000b). This construct contains the Renilla luciferase gene downstream of an SV40 promoter and the firefly luciferase gene downstream of a cloning site that contains EcoRI and NcoI restriction sites. It was also cloned into pBR (Mitchell et al, 2005), which lacks a viral promoter. Constructs designated pRGasF and pBRGas were generated. Constructs containing the c-myc IRES (pRMF) and pBRMyc were used as controls (Stoneley et al, 2000b).

\section{Transfections}

Transfections were carried out using Lipofectamine 2000 (Invitrogen, Paisley, UK). Test plasmids were co-transfected with a control plasmid (Coldwell et al, 2000), encoding $\beta$-galactosidase $(\beta$-gal) to provide a transfection control, using a ratio of 5:1, test 
plasmid: $\beta$-gal plasmid. Following initial optimisation experiments $500 \mathrm{ng}$ of plasmid was used to transfect $2 \times 10^{5}$ cells in 24-well plates. A $500 \mathrm{ng}$ aliquot of test plasmid plus $100 \mathrm{ng}$ of $\beta$-gal plasmid were diluted to $50 \mu \mathrm{l}$ in Optimem (Gibco BRL), mixed with $2 \mu \mathrm{l}$ Lipofectamine 2000 (diluted to $50 \mu \mathrm{l}$ in Optimem) for $20 \mathrm{~min}$ at room temperature, and added to cells in $500 \mu \mathrm{l}$ culture medium. To measure basal IRES activity, cells were incubated for $24 \mathrm{~h}$ before harvesting. To investigate the activity of the IRES following treatment with mitomycin C (MMC; Sigma) or exposure to hypoxia, medium was changed $6 \mathrm{~h}$ after transfection and either normal culture medium, medium containing 2-20 $\mu \mathrm{g} \mathrm{ml}^{-1} \mathrm{MMC}$ or serum-free medium was added. The cells were then returned to the $\mathrm{CO}_{2}$ incubator or exposed to hypoxia ( $1 \%$ oxygen, $5 \% \mathrm{CO}_{2}$, $37^{\circ} \mathrm{C}$ ) using an Invivo2 400 Hypoxia workstation (Biotrace Fred Baker, Bridgend, UK) for a further $24 \mathrm{~h}$.

Dual transfection of plasmids and siRNAs was carried out by preparing the plasmid transfection mix as above but in half the volume, and preincubating $500 \mathrm{ng}$ of siRNA with $1 \mu \mathrm{l}$ siPortAmine (Invitrogen) in $25 \mu \mathrm{l}$ serum-free medium. The two transfection reagents were then mixed immediately before addition to the cells. The Renilla siRNA had the target sequence AACGCGGCCTC TTCTTATTTA, which was designed using the Qiagen siRNA Design tool and has a perfect match with Renilla luciferase, but nine mismatches and a maximum of six adjacent matching nucleotides when aligned with the sequence of firefly luciferase.

\section{Reporter assays}

Cells were harvested and analysed using the Stop and Glo luciferase assay (Promega). Cells were lysed in PLB buffer (Promega) and both firefly and Renilla luciferase activity measured using a MicroLumi XS luminometer (Harta Instruments, Gaithersburg, Maryland, USA). $\beta$-Gal activity was measured using the Galacto-Light Plus system (ABI). All luciferase readings were expressed relative to the $\beta$-gal activity in the same sample.

\section{Statistics}

The Student $t$-test was used to compare luciferase activity in cells transfected with IRES constructs $v s$ control vectors or exposure to hypoxia $v s$ normoxia. The effect of treatment with increasing concentrations of MMC was analysed using one-way ANOVA with Dunnett's multiple comparison test, with $P<0.05$ considered significant.

\section{RESULTS}

\section{The gastrin transcript identified by RLM-RACE does not contain the $5^{\prime}$ UTR of the transcript defined by Ensembl}

The sequence of the $5^{\prime}$ UTR of gastrin transcripts isolated from the pancreatic cell line, PAN1, was analysed by RLM-RACE using two gastrin-specific primers, one in each round of PCR, paired with primers specific for the $5^{\prime}$ adapter sequence. The PCR products were cloned and eight individual clones sequenced. Three unique clones were identified (1,2 and 7); the remaining clones were identical to clone 2 . The sequences of clones 1,2 and 7 were aligned with the gastrin genomic sequence, the sequence of the $5^{\prime}$ UTR of the gastrin transcript provided by Ensembl and the 5'UTR of the transcript described by Kato et al (1983) (GenBank entry, X00183). The alignment is shown in Figure 1. There was only a small region of high homology with the $5^{\prime}$ UTR of the Ensembl transcript consisting of 10 nucleotides (indicated in Figure 1 by $\sim$ ) derived from the end of exon 1 ( 5 nucleotides) and the beginning of exon 2 (5 nucleotides). However, there was a high degree of homology with the sequence defined by Ensembl as intron 1 and the $5^{\prime}$ UTR of the transcript defined in GenBank entry X00183, with differences only at two positions (indicated by $\wedge$ in Figure 1); the

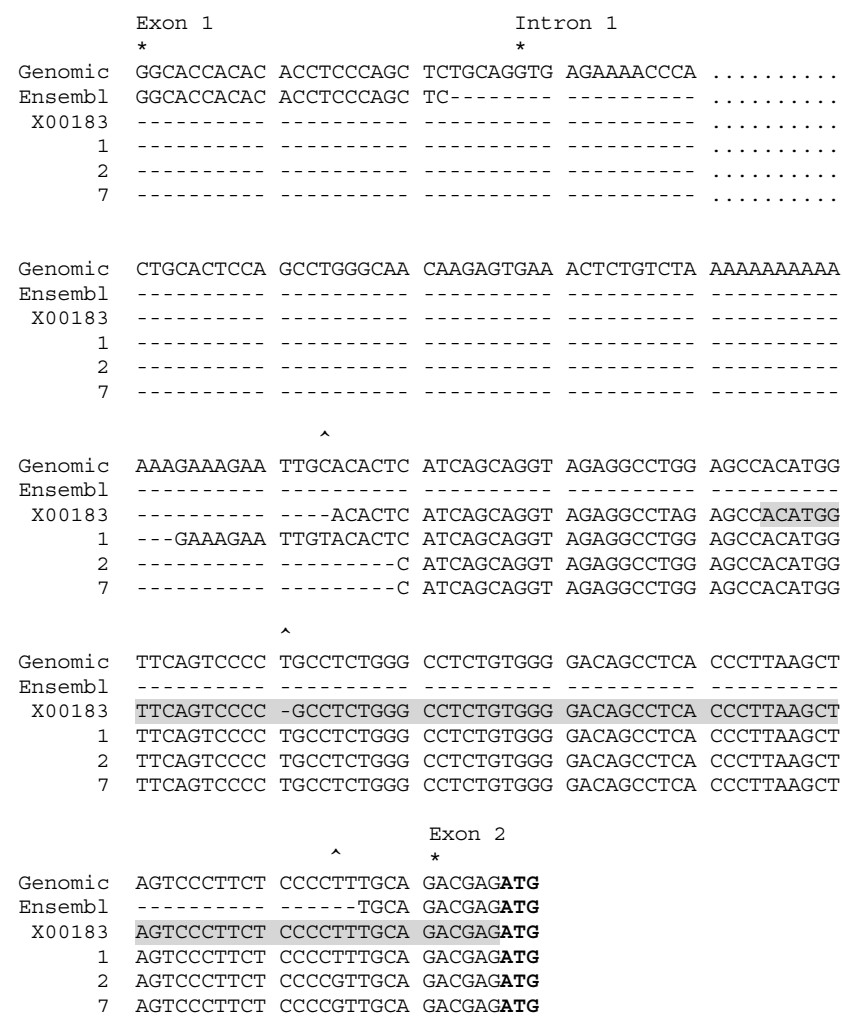

Figure I Alignment of Ensembl gastrin genomic sequence (Genomic) Ensembl gastrin transcript (Ensembl), GenBank sequence X00/83 (X00I83) and three representative RLM-RACE clones (I, 2 and 7). Clones I and 7 occurred once each, and clone 2 occurred six times. The beginning of exon I, intron I and exon 2 are indicated by * and the ATG start codon is highlighted in bold. The central $2860 \mathrm{bp}$ of intron I have been omitted for clarity and their position is indicated by -...-.--; only sequence of the first 13-bp and final 171-bp of intron I are shown. The RLM-RACE clones have high homology with the region defined by Ensembl

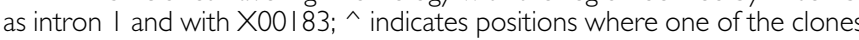
or $\mathrm{X00I83} \mathrm{did} \mathrm{not} \mathrm{match} \mathrm{the} \mathrm{genomic} \mathrm{sequence.} \mathrm{There} \mathrm{were} \mathrm{only} 10$ nucleotides, which matched the Ensembl transcript, indicated by $\sim$ below the sequence. The UTRScan-predicted IRES within X00I83 is highlighted by shading.

Ensembl genomic sequence and the RLM-RACE clones all have a $\mathrm{T}$ at position -88 , which is missing in $\mathrm{X} 00183$ and, in addition, the RLM-RACE clones represented by clones 2 and 7 have a G/T substitution at -66 . For six of the clones (represented by clone 2 in Figure 1) and in clone 7 , the sequence started at a point $106 \mathrm{bp}$ upstream of the ATG codon; for clone 1 the start point was at -122 . Thus, the transcript identified by RLM-RACE in the PAN1 cells is different from the gastrin transcript described in Ensembl and more closely resembles that described in GenBank entry, X00183.

\section{The transcript identified by RLM-RACE is expressed in a range of GI cancer cells}

RT-PCR was carried out in a panel of GI cancer cells using primers specific for the $5^{\prime}$ UTR of the Ensembl transcript or the alternative transcript in combination with a common primer (Figure 2). Both transcripts were present in all the cell lines tested, which included cells of pancreatic, colonic, gastric and oesophageal origin. For the alternative transcript, two amplification products were observed: a band of the predicted size of $360 \mathrm{bp}$ in all of the cell lines except for 

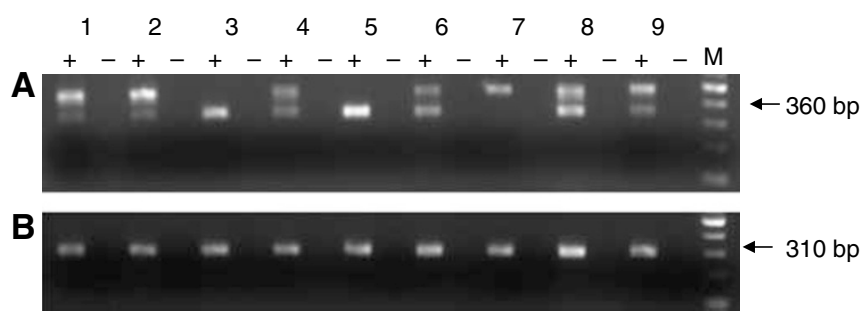

Figure 2 Reverse transcriptase-PCR to investigate expression of the two gastrin transcripts in a panel of GI cancer cell lines: (I) PANI, (2) BXPC3 (pancreatic); (3) HCTI I6, (4) HT29 (5) CI70HM2 (colonic); (6) STI 6, (7) MGLVAI (gastric); (8) OEI9 and (9) OE2I (oesophageal). cDNA $(+)$ and a negative control in which the RT was omitted $(-)$ were amplified using a primer specific for each transcript $((\mathbf{A})$ alternative transcript and (B) Ensembl transcript) and a common primer. The expected position of the bands is indicated by an arrow based on the position of the 100-bp markers (M).

MGLVA1 cells and a higher molecular weight product of $490 \mathrm{bp}$ in all of the cell lines except HCT116 and C170HM2 cells. The size of this larger product equates to the expected transcript but with the intron 2 retained. However, this does not appear to be derived from amplification of contaminating genomic DNA as it did not appear in the RT-negative control.

\section{Prediction of an IRES within the gastrin transcript identified by RLM-RACE}

The $5^{\prime}$ UTR of the transcript identified in the GI cancer cells by RLM-RACE is GC-rich, with a $60.7 \%$ GC content and includes an AUG upstream of the translation start site. Therefore, we analysed the sequence for elements that might regulate the stability of the transcript or its translation. The sequences of the $5^{\prime}$ UTRs of the gastrin transcript identified by RLM-RACE, the Ensembl transcript and the transcript described in X00183 were submitted to UTRScan. An IRES, consisting of $91 \mathrm{bp}$ of sequence upstream of the start codon, was predicted to be present only in the X00183 gastrin transcript (sequence shaded in Figure 1), in spite of the homology between the X00183 transcript and that identified by RLM-RACE, the RLM-RACE transcript differing only by the inclusion of one additional $\mathrm{T}$ at -66 .

\section{Assessment of the activity of the putative IRES to regulate translational activity}

To assess the ability of the putative IRES to regulate translation a bicistronic reporter vector, pRF was used, consisting of two luciferase genes encoded within a single transcript expressed under the control of an SV40 promoter. The predicted IRES was amplified and cloned into pRF to generate a construct pRGasF. The sequence of two clones was compared with the sequence of the transcript predicted in X00183. The additional $\mathrm{T}$ nucleotide at position -66 was found to be present in both clones, as expected from the RLM-RACE data; in addition, some minor differences were found at positions $-1 / 2$ and $-96 / 97$.

The $5^{\prime}$ Renilla luciferase in the bicistronic vector was designed so it would be translated in a cap-dependent manner but translation of the $3^{\prime}$ firefly luciferase would be dependent on the insertion of an IRES. For each construct, the activity of the IRESdependent luciferase was measured relative to the empty vector and cap-dependent luciferase signal in Pan1 and HCT116 cells. There were significant 8 - and 15-fold increases $(P=0.02$ and $P<0.001)$ in luciferase activity in PAN1 and HCT116 cells, respectively, following insertion of the putative gastrin IRES (pRGasF). A construct containing the c-myc IRES (pRMF) was included as a positive control in these experiments (Figure $3 \mathrm{~A}$ and B). The activity of this IRES was particularly high in PAN1 cells with a 49 -fold induction of activity $(P=0.014)$ but, in HCT116 cells, the activity was similar to that of the gastrin IRES, with a 10 -fold increase compared with the empty vector, $\mathrm{pRF}(P=0.007)$.

\section{The putative gastrin IRES has no activity in a plasmid lacking a promoter}

To verify that the putative gastrin IRES is not acting as a promoter in $\mathrm{pRGasF}$, it was cloned into a reporter vector, $\mathrm{pBR}$, lacking a viral promoter but with viral enhancer elements still present downstream of the firefly luciferase. There was no increase in luciferase activity in the $\mathrm{pBR}$ construct when the putative gastrin IRES was inserted (pBRGas), suggesting that the sequence is not able to act as a promoter, even in the presence of viral enhancer sequences while activity of the pRGasF IRES construct was as expected (Figure 3C). In addition, when a Renilla siRNA was used to treat the pRGasF-transfected cells, there was a significant knockdown of the firefly luciferase activity in parallel with the knockdown of the Renilla activity $(P<0.005$ for both), suggesting that both are encoded on the same transcript (Figure 3D), and RT-PCR of RNA from pRGasF-transfected cells using primers located within the Renilla and firefly luciferases, respectively, revealed a single band of approximately $300 \mathrm{bp}$ (Figure 3E), which equates to a full-length RNA without smaller splice variants.

\section{The activity of the putative gastrin IRES is upregulated following exposure of the cells to genotoxic stress or hypoxia}

Since a role for gastrin in promoting cell survival has been shown, the activity of the IRES was studied after exposure of HCT116 and PAN1 cells transfected with pRGasF to a range of concentrations of the DNA-damaging agent, MMC. As expected, with increasing concentrations of MMC, activity of the cap-dependent luciferase was switched off (Figure 4A). However, activity of the IRES-driven luciferase was either maintained or increased resulting in an increase in the ratio of firefly to Renilla luciferase activity in both cell lines (Figure 4B and C). This suggests that the putative gastrin IRES is able to support translation under circumstances where cap-dependent translation is not functional.

Gastrin is also known to promote angiogenesis, and so we investigated the effect of hypoxia on IRES-driven luciferase activity. The effect of serum was incorporated into this study as the activity of the HIF IRES has previously been shown to be most active in serum-free conditions. In HCT116 cells, there was a significant increase in the relative activity of the IRES-driven compared with the cap-driven luciferase after $24 \mathrm{~h}$ exposure to hypoxia in the presence of serum $(P=0.016)$. There was also an increase in the absence of serum, but this did not reach statistical significance $(P=0.078)$.

\section{DISCUSSION}

We have shown that a gastrin transcript expressed in a panel of GI cancer cell lines contains an IRES that has basal activity in both pancreatic and colon cancer cells. The sequence appears to be acting as a true IRES, as activity is lost in the absence of a viral promoter. Under conditions of cellular stress, activity of the IRES is increased or maintained at higher levels than cap-dependent translation suggesting that it is controlled by a distinct mechanism, as shown for the c-myc IRES (Subkhankulova et al, 2001).

The IRES-containing transcript found in the GI cancer cell lines appears to use a transcription start site within the sequence assigned by Ensembl as intron 1 and is homologous to the transcript described in G cells (Kato et al, 1983). These authors localised a possible transcription start site by S1 nuclease mapping, 


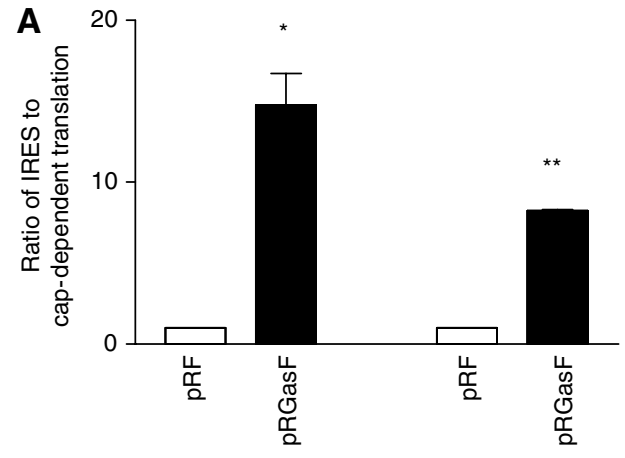

PAN1

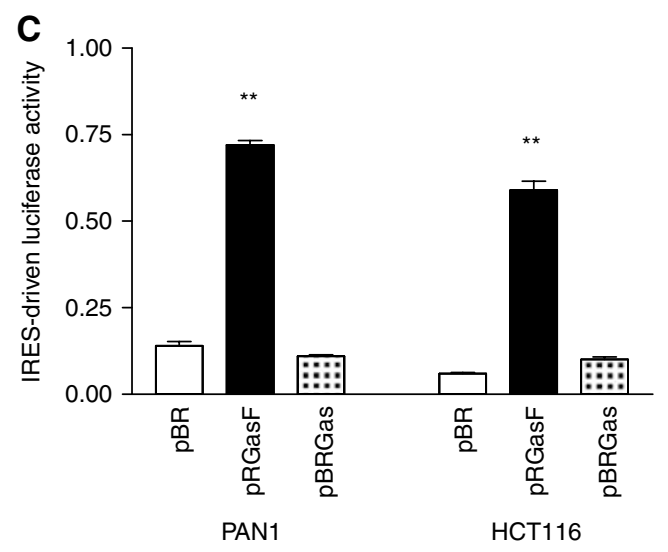

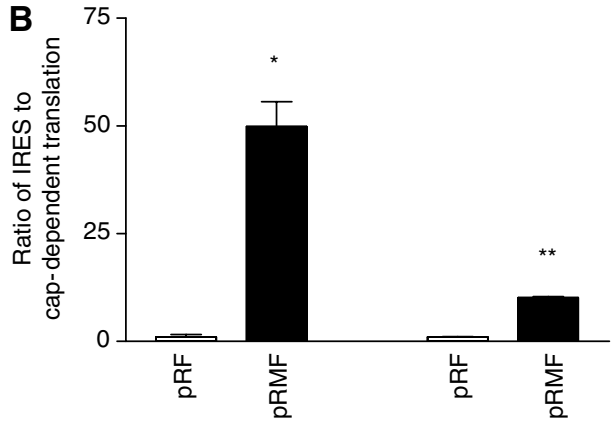

PAN 1

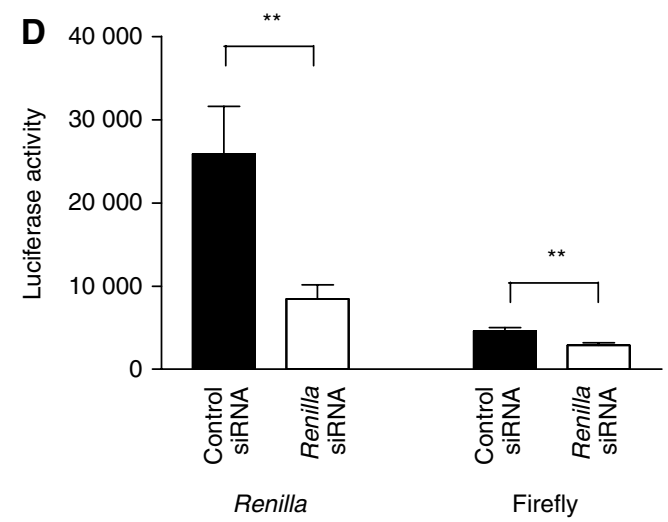

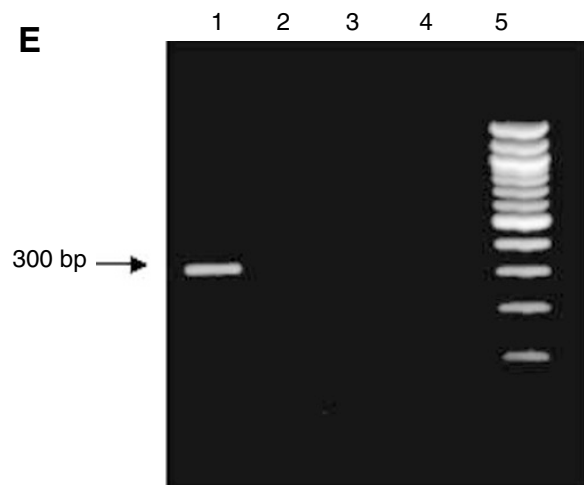

Figure 3 Activity of the putative gastrin IRES and Myc IRES in pancreatic (PANI) and colon (HCTI I6) cancer cell lines. Basal activity of (A) the gastrin IRES (pRGasF) or (B) Myc IRES (pRMF) compared with empty vector (pRF). (C) Activity of the gastrin IRES in a promotorless plasmid (pBRGas) compared with the empty vector (pBR) or pRGasF. (D) Significant knockdown of both Renilla and firefly luciferase activity in HCTI I 6 cells transfected with pRGasF and a Renilla siRNA, compared with cells transfected with pRGasF and a control siRNA ( $P<0.005$ for both). (E) Reverse transcriptase-PCR of cells transfected with pRGasF using forward and reverse primers located within the Renilla and firefly luciferase sequences. A single band of $\sim 300$ bp was observed (Lane I). No bands were observed in the RT-negative, cDNA reagent and RNA isolation controls (Lanes 2-4). Lane 5: markers. Statistical significance is indicated by $* P<0.05$ or $* * * 0.01$

following hybridisation with gastric antrum polyA RNA, to approximately $111 \mathrm{bp}$ upstream of the start codon. Potential Goldberg-Hogness and CCAAT boxes were found upstream of the transcription start site in the $5^{\prime}$ flanking DNA sequence (Kato et al, 1983).

Seven of the independent clones generated by RLM-RACE started $106 \mathrm{bp}$ upstream of the ATG start codon and the remaining clone started a further $16 \mathrm{bp}$ upstream. It is unlikely that the products arise from amplification of DNA fragments; such fragments arise at random and are unlikely to have $5^{\prime}$ ends, which cluster at a single location. In addition, RLM-RACE is designed to prevent amplification of DNA templates; it includes steps which remove free $5^{\prime}$ phosphates from uncapped RNAs and contaminating DNA, preventing ligation of the adapter to such nucleic acids, and no product was detected when the enzyme TAP, which removes the cap to reveal the free $5^{\prime}$ phosphate on mRNA and allow ligation of the adapter, was omitted.

In addition, using RT-PCR, the alternative transcript was shown to be present in all of the GI cancer cell lines investigated including cells of pancreatic, colonic, gastric and oesophageal origin. An amplification product with a higher molecular weight than that predicted was also amplified in most of the GI cancer cell panel using a primer specific for the alternative transcript; this appears to have retained intron 2 and further investigation will be required to determine how this transcript arises and whether it is functional. However, the predicted transcript was also present in the majority of the cell lines investigated; while the intensity of the band derived from the Ensembl transcript was fairly uniform 

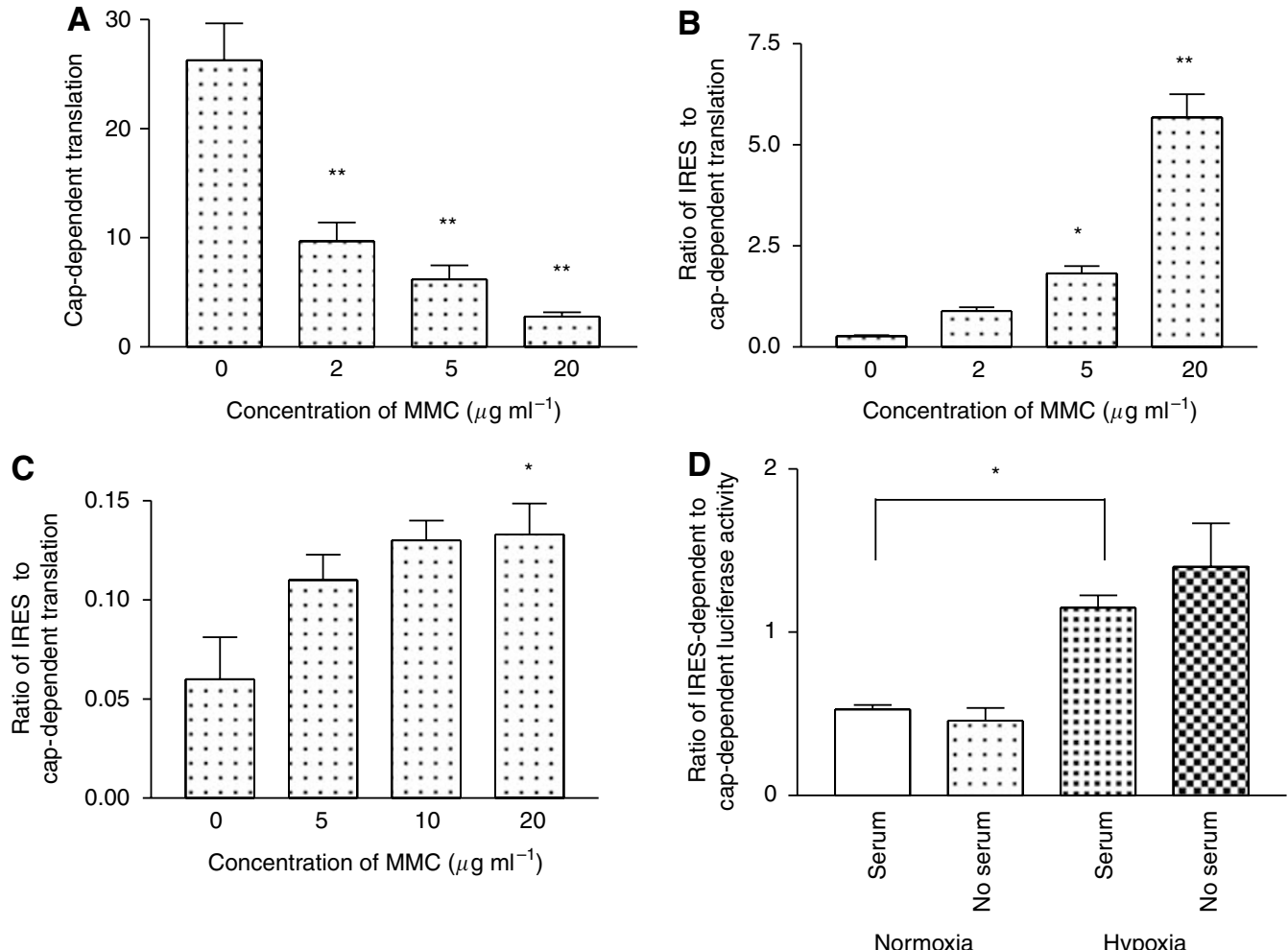

Figure 4 Effect of MMC and hypoxia on IRES activity. Treatment with MMC significantly reduced cap-dependent translation of the Renilla luciferase (A) but resulted in an increase in the ratio of IRES-dependent to cap-dependent translation in both HCTI I6 (B) and PANI cells (C). Similarly, $24 \mathrm{~h}$ exposure to a hypoxic environment increased IRES-dependent translation (D). Statistical significance is indicated by $* P<0.05$ or $* * P<0.01$.

across the cell line panel, the intensity of the bands from the alternative transcript varied between cell lines.

This raises the question of how transcription of these two mRNAs is regulated. Most studies investigating regulation of gastrin gene transcription in GI cancer cells have used constructs based on the promoter associated with the Ensembl transcript. Further studies to investigate the DNA flanking sequence will be required to identify the promoter, that drives transcription of this alternative mRNA. Transcription from alternative start sites has been shown previously in certain genes, including oncogenes, giving rise to transcripts that are differentially regulated at the translational level; for example, $c-m y c$ has four alternative transcripts driven by different promoters and only one of these contains an IRES (Marcu et al, 1992).

The $5^{\prime}$ UTR of the alternative gastrin transcript described in the present study is GC-rich, in keeping with other described IRESs, which are generally highly structured (Pickering and Willis, 2005). Since the function of IRESs is probably dependent on their threedimensional structure rather than the presence of specific sequences, it has been difficult to identify motifs that allow their definitive identification. A recent paper has identified a motif, $(\mathrm{CCU})_{n}$ occurring within a polypyrimidine tract, which acts as an artificial IRES when the polypyrimidine tract protein, one of the trans-acting factors thought to be involved in initiation of translation from IRESs, is present (Mitchell et al, 2005). We used a bioinformatics-based method to look for common functional sequences that occur in the UTRs of transcripts (Pesole and Liuni, 1999), which includes a search for a motif common to IRESs of cellular RNAs that forms a Y-type stem loop structure (Le and Maizel, 1997). Interestingly, while UTRScan identified a potential 94-bp IRES within the $5^{\prime}$ UTR of the transcript described in GenBank entry X00183, it did not identify an IRES within the $5^{\prime}$ UTR of the transcript identified by RLM-RACE, which differs only by insertion of a single nucleotide $66 \mathrm{bp}$ upstream of the start codon, showing that such programs can only be used to provide guidance.

Therefore, we tested the ability of the predicted IRES to drive translation within a bicistronic reporter construct (Stoneley et al, 1998; Coldwell et al, 2001; Mitchell et al, 2001). There was significant IRES-dependent activity in both of the GI cancer cell lines used. In PAN1 cells, the IRES-driven luciferase activity was lower than that observed using a construct containing the c-myc IRES. In HCT116 cells, while the activity of the gastrin IRES was lower than in PAN1 cells, it was higher than that of the c-myc IRES in these cells. For these experiments, we inserted only the 94-bp sequence, which was predicted to be an IRES by UTRScan and used the sequence variant found in the PAN1 gastrin transcript. It is possible that the removal of the inserted $\mathrm{T}$ and use of the whole of the transcript's 5'UTR might lead to a higher level of activity.

The luciferase activity observed when the gastrin $5^{\prime}$ UTR sequence was inserted into the bicistronic construct might occur because the sequence is able to act as a promoter and allow independent transcription of the downstream luciferase. However, when the gastrin sequence was cloned upstream of luciferase in a vector that did not contain a promoter, there was no luciferase activity compared with the empty vector. This is further suggested by the reduction in firefly luciferase activity observed in parallel with the reduction in Renilla luciferase activity following treatment with a Renilla luciferase-specific siRNA. Another possible explanation for the activity of the luciferase downstream of the putative gastrin IRES is that the sequence acts as a splice acceptor, as has been suggested for the XIAP sequence (Van Eden et al, 2004), but we did not observe any smaller RT-PCR products to indicate that the IRES sequence was being removed by splicing. Taken together, these data suggest that the gastrin sequence is acting as an IRES. 
Under conditions of cellular stress, for example apoptosis, capdependent translation is switched off as a result of cleavage of initiation factors such as EIF4G and EIF4B, which prevents circularisation of the transcript (Spriggs et al, 2005). Expression of genes that play an anti-apoptotic role in cancer has been shown to be maintained via use of an IRES (Coldwell et al, 2001), and since such a role has also been ascribed to gastrin in GI cancer cells (Harris et al, 2004), we investigated whether the gastrin IRES was active during apoptosis induced by the genotoxic agent, MMC. Gastrin also has a role in promoting angiogenesis and so, in parallel, we investigated the effect of hypoxia on the gastrin IRES activity. In both cases, activity of the IRES was increased relative to cap-dependent translation.

The translation initiation factors that regulate expression from IRESs have begun to be elucidated. They appear to include canonical translation initiation factors such as eIF2, eIF3 and eIF4F (Pestova et al, 1996) and also other trans-acting factors, and may act to change the secondary structure of the IRES to facilitate ribosomal binding (Mitchell et al, 2003; Pickering et al, 2004).
Differential expression of such factors may explain the different patterns of activity of individual IRESs during development and differentiation (Sella et al, 1999; Creancier et al, 2000; Mitchell et al, 2003) and in cancer (Evans et al, 2003). In the present study, the basal activity of the $c-m y c$ and gastrin IRESs in PAN1 and HCT116 cells, which are of pancreatic and colonic origin, did not correlate. In addition, the activity of the $c$-myc IRES, which has previously been shown to be upregulated by MMC in HeLa cells (Subkhankulova et al, 2001), was not increased following exposure of these GI cancer cells to MMC, but it was responsive to hypoxia (data not shown). Taken together, these data suggest that expression from the two IRESs is controlled independently, involving different trans-acting factors.

Expression of a transcript containing an IRES, which allows continued expression of gastrin peptides in the face of apoptotic stimuli or hypoxia would promote the survival of GI cancer cells. Thus, further investigation of the mechanisms regulating expression of the transcript and activity of the IRES in different cell types is warranted.

\section{REFERENCES}

Beales IL (2004) Gastrin and interleukin-1beta stimulate growth factor secretion from cultured rabbit gastric parietal cells. Life Sci 75: 2983-2995

Bushell M, Stoneley M, Sarnow P, Willis AE (2004) Translation inhibition during the induction of apoptosis: RNA or protein degradation? Biochem Soc Trans 32: 606-610

Clarke PA, Dickson JH, Harris JC, Grabowska A, Watson SA (2006) Gastrin enhances the angiogenic potential of endothelial cells via modulation of heparin-binding epidermal-like growth factor. Cancer Res 66: $3504-3512$

Coldwell MJ, deSchoolmeester ML, Fraser GA, Pickering BM, Packham G, Willis AE (2001) The p36 isoform of BAG-1 is translated by internal ribosome entry following heat shock. Oncogene 20: 4095-4100

Coldwell MJ, Mitchell SA, Stoneley M, MacFarlane M, Willis AE (2000) Initiation of Apaf-1 translation by internal ribosome entry. Oncogene 19: $899-905$

Creancier L, Morello D, Mercier P, Prats AC (2000) Fibroblast growth factor 2 internal ribosome entry site (IRES) activity ex vivo and in transgenic mice reveals a stringent tissue-specific regulation. J Cell Biol 150: $275-281$

Evans JR, Mitchell SA, Spriggs KA, Ostrowski J, Bomsztyk K, Ostarek D, Willis AE (2003) Members of the poly ( $\mathrm{rC}$ ) binding protein family stimulate the activity of the c-myc internal ribosome entry segment in vitro and in vivo. Oncogene 22: 8012-8020

Ferrand A, Wang TC (2006) Gastrin and cancer: a review. Cancer Lett 238: $15-29$

Goetze JP, Nielsen FC, Burcharth F, Rehfeld JF (2000) Closing the gastrin loop in pancreatic carcinoma: coexpression of gastrin and its receptor in solid human pancreatic adenocarcinoma. Cancer 88: 2487-2494

Grabowska AM, Hughes J, Watson SA (2007) Use of interfering RNA to investigate the role of endogenous gastrin in the survival of gastrointestinal cancer cells. Br J Cancer 96: 464-473

Harris JC, Clarke PA, Awan A, Jankowski J, Watson SA (2004) An antiapoptotic role for gastrin and the gastrin/CCK-2 receptor in Barrett's esophagus. Cancer Res 64: 1915-1919

Holcik M, Sonenberg N (2005) Translational control in stress and apoptosis. Nat Rev Mol Cell Biol 6: 318-327

Hur K, Kwak MK, Lee HJ, Park do J, Lee HK, Lee HS, Kim WH, Michaeli D, Yang HK (2006) Expression of gastrin and its receptor in human gastric cancer tissues. I Cancer Res Clin Oncol 132: 85-91

Ito R, Sato K, Helmer T, Jay G, Agarwal K (1984) Structural analysis of the gene encoding human gastrin: the large intron contains an Alu sequence. Proc Natl Acad Sci USA 81: $4662-4666$

Kato K, Hayashizaki Y, Takahashi Y, Himeno S, Matsubara K (1983) Molecular cloning of the human gastrin gene. Nucleic Acids Res 11: $8197-8203$

Koh TJ, Bulitta CJ, Fleming JV, Dockray GJ, Varro A, Wang TC (2000) Gastrin is a target of the beta-catenin/TCF-4 growth-signaling pathway in a model of intestinal polyposis. J Clin Invest 106: 533-539
Konturek PC, Kania J, Kukharsky V, Ocker S, Hahn EG, Konturek SJ (2003) Influence of gastrin on the expression of cyclooxygenase-2, hepatocyte growth factor and apoptosis-related proteins in gastric epithelial cells. J Physiol Pharmacol 54: 17-32

Le SY, Maizel Jr JV (1997) A common RNA structural motif involved in the internal initiation of translation of cellular mRNAs. Nucleic Acids Res 25 $362-369$

Marcu KB, Bossone SA, Patel AJ (1992) myc function and regulation. Annu Rev Biochem 61: 809-860

McWilliams DF, Watson SA, Crosbee DM, Michaeli D, Seth R (1998) Coexpression of gastrin and gastrin receptors (CCK-B and delta CCK-B) in gastrointestinal tumour cell lines. Gut 42: 795-798

Merchant JL, Demediuk B, Brand SJ (1991) A GC-rich element confers epidermal growth factor responsiveness to transcription from the gastrin promoter. Mol Cell Biol 11: 2686-2696

Miller DL, Dibbens JA, Damert A, Risau W, Vadas MA, Goodall GJ (1998) The vascular endothelial growth factor mRNA contains an internal ribosome entry site. FEBS Lett 434: 417-420

Mitchell SA, Brown EC, Coldwell MJ, Jackson RJ, Willis AE (2001) Protein factor requirements of the Apaf-1 internal ribosome entry segment: roles of polypyrimidine tract binding protein and upstream of N-ras. Mol Cell Biol 21: $3364-3374$

Mitchell SA, Spriggs KA, Bushell M, Evans JR, Stoneley M, Le Quesne JP, Spriggs RV, Willis AE (2005) Identification of a motif that mediates polypyrimidine tract-binding protein-dependent internal ribosome entry. Genes Dev 19: $1556-1571$

Mitchell SA, Spriggs KA, Coldwell MJ, Jackson RJ, Willis AE (2003) The Apaf- 1 internal ribosome entry segment attains the correct structural conformation for function via interactions with PTB and unr. Mol Cell 11: $757-771$

Mukawa K, Fujii S, Takeda J, Kitajima K, Tominaga K, Chibana Y, Fujita M, Ichikawa K, Tomita S, Ono Y, Imura J, Kawamata H, Chiba T, Hiraishi H, Terano A, Fujimori T (2005) Analysis of K-ras mutations and expression of cyclooxygenase- 2 and gastrin protein in laterally spreading tumors. J Gastroenterol Hepatol 20: 1584-1590

Nakata H, Wang SL, Chung DC, Westwick JK, Tillotson LG (1998) Oncogenic ras induces gastrin gene expression in colon cancer. Gastroenterology 115: 1144-1153

Pesole G, Liuni S (1999) Internet resources for the functional analysis of $5^{\prime}$ and $3^{\prime}$ untranslated regions of eukaryotic mRNAs. Trends Genet 15: 378

Pestova TV, Hellen CU, Shatsky IN (1996) Canonical eukaryotic initiation factors determine initiation of translation by internal ribosomal entry. Mol Cell Biol 16: 6859-6869

Pickering BM, Mitchell SA, Spriggs KA, Stoneley M, Willis AE (2004). Bag-1 internal ribosome entry segment activity is promoted by structural changes mediated by poly $(\mathrm{rC})$ binding protein 1 and recruitment of polypyrimidine tract binding protein 1. Mol Cell Biol 24: $5595-5605$ 
Pickering BM, Willis AE (2005) The implications of structured $5^{\prime}$ untranslated regions on translation and disease. Semin Cell Dev Biol 16: 39-47

Ramamoorthy S, Stepan V, Todisco A (2004) Intracellular mechanisms mediating the anti-apoptotic action of gastrin. Biochem Biophys Res Commun 323: $44-48$

Rehfeld JF, Bardram L, Hilsted L (1989) Gastrin in human bronchogenic carcinomas: constant expression but variable processing of progastrin. Cancer Res 49: 2840 - 2843

Rieder G, Merchant JL, Haas R (2005) Helicobacter pylori cag-type IV secretion system facilitates corpus colonization to induce precancerous conditions in Mongolian gerbils. Gastroenterology 128: 1229-1242

Sella O, Gerlitz G, Le SY, Elroy-Stein O (1999) Differentiation-induced internal translation of c-sis mRNA: analysis of the cis elements and their differentiation-linked binding to the hnRNP C protein. Mol Cell Biol 19: $5429-5440$

Sontheimer EJ, Carthew RW (2005) Silence from within: endogenous siRNAs and miRNAs. Cell 122: 9-12

Spriggs KA, Bushell M, Mitchell SA, Willis AE (2005) Internal ribosome entry segment-mediated translation during apoptosis: the role of IREStrans-acting factors. Cell Death Differ 12: 585-591

Stoneley M, Chappell SA, Jopling CL, Dickens M, MacFarlane M, Willis AE (2000a) c-Myc protein synthesis is initiated from the internal ribosome entry segment during apoptosis. Mol Cell Biol 20: $1162-1169$

Stoneley M, Paulin FE, Le Quesne JP, Chappell SA, Willis AE (1998) C-Myc $5^{\prime}$ untranslated region contains an internal ribosome entry segment. Oncogene 16: $423-428$
Stoneley M, Subkhankulova T, Le Quesne JP, Coldwell MJ, Jopling CL, Belsham GJ, Willis AE (2000b) Analysis of the c-myc IRES; a potential role for cell-type specific trans-acting factors and the nuclear compartment. Nucleic Acids Res 28: 687-694

Subkhankulova T, Mitchell SA, Willis AE (2001) Internal ribosome entry segment-mediated initiation of $\mathrm{c}-\mathrm{Myc}$ protein synthesis following genotoxic stress. Biochem J 359: $183-192$

Suzuki T, Grand E, Bowman C, Merchant JL, Todisco A, Wang L, Del Valle J (2001) TNF-alpha and interleukin 1 activate gastrin gene expression via MAPK- and PKC-dependent mechanisms. Am J Physiol Gastrointest Liver Physiol 281: G1405-G1412

Vagner S, Gensac MC, Maret A, Bayard F, Amalric F, Prats $H$, Prats AC (1995) Alternative translation of human fibroblast growth factor 2 mRNA occurs by internal entry of ribosomes. Mol Cell Biol 15: $35-44$

Van Eden ME, Byrd MP, Sherrill KW, Lloyd RE (2004) Demonstrating internal ribosome entry sites in eukaryotic mRNAs using stringent RNA test procedures. RNA 10: $720-730$

van Solinge WW, Odum L, Rehfeld JF (1993) Ovarian cancers express and process progastrin. Cancer Res 53: $1823-1828$

Watson SA, Grabowska AM, El-Zaatari M, Takhar A (2006) Gastrin - active participant or bystander in gastric carcinogenesis? Nat Rev Cancer 6: 936- 946

Wiborg O, Berglund L, Boel E, Norris F, Norris K, Rehfeld JF, Marcker KA, Vuust J (1984) Structure of a human gastrin gene. Proc Natl Acad Sci USA 81: 1067 - 1069 